\title{
Comparison of Percutaneous Transforaminal Endoscopic Discectomy with and without Epidural Steroid Application in the Treatment of Lumbar Disc Herniation: A Minimum of 2 Years of Follow-Up
}

\author{
Jianhua $\mathrm{LV}^{1}$, Xiang WANG ${ }^{2}$, Meihua $\mathrm{CHEN}^{2}$, Jianfei $\mathrm{WU}^{2}$ \\ ${ }^{1}$ The Affiliated Hospital of Putian University, Department of Orthopaedics, Putian, Fujian, China \\ ${ }^{2}$ The 907 Hospital of Joint Logistic Support Force, Department of Orthopaedics, Nanping, Fujian, China
}

Corresponding author: Jianfei WU Wujianfei89007@163.com

\section{ABSTRACT}

AIM: To compare the efficacy of percutaneous transforaminal endoscopic discectomy with and without epidural steroid application in the treatment of lumbar disc herniation.

MATERIAL and METHODS: A total of 101 patients who had indications for percutaneous transforaminal endoscopic discectomy were retrospectively reviewed. Patients were divided into two groups based on whether epidural steroids were applied following the surgical procedure. Each patient in group A received an epidural injection of $40 \mathrm{mg}$ triamcinolone acetonide through the working sleeve following the surgical procedure. However, patients in group B were not given the drug. Preoperative and postoperative radicular pain was measured with the visual analogue scale (VAS). Functional and satisfaction outcomes were measured with the Oswestry Disability Index (ODI) and the modified MacNab criteria, respectively.

RESULTS: A total of 97 patients had a complete follow-up of longer than two years without recurrence. The sample sizes of group A and group B were 56 and 41 . The VAS scores and ODI scores at each postoperative follow-up point were significantly lower than the preoperative values $(p<0.001)$. However, there were no significant between-group differences $(p>0.05)$. The secondary outcomes of the changes in the VAS and ODI scores were also equivalent between the groups at the 3-month and 2-year follow-ups ( $p>0.05$ ). No significant difference was observed in the modified MacNab criteria between the groups at 2 years $(p=0.7715)$.

CONCLUSION: Percutaneous transforaminal endoscopic discectomy is a safe and effective minimally invasive surgery for radiculopathy caused by lumbar disc herniation. Epidural steroid injection following the surgical procedure offered no benefit compared with surgery alone.

KEYWORDS: Lumbar disc herniation, Transforaminal, Endoscopic discectomy, Epidural injections, Steroids

\section{INTRODUCTION}

$\mathrm{P}$ ercutaneous transforaminal endoscopic discectomy (PTED), one of the least invasive surgeries for lumbar disc herniation, has been widely used at present. Its clinical advantages have been well recognized by spine surgeons. It has the same decompression efficacy for the affected nerve roots as microendoscopic discectomy (MED) (5). However, surgical decompression may sometimes fail to ameliorate symptoms because of nerve root inflammation, which determines the extent and severity of pain in those patients (22). Nerve root inflammation, also known as
Jianhua LV (1) : 0000-0003-4538-6181 Xiang WANG (1) : 0000-0001-6012-5241
Meihua CHEN (1) : 0000-0001-9501-076X

Jianfei WU (D) : 0000-0002-3643-9192 
radiculitis, can be eliminated by epidural steroid injection, one of the most common nonsurgical interventions for radicular pain caused by lumbar disc herniation $(6,13)$. Thus far, it is unclear whether it is possible to further improve the clinical efficacy by injecting steroids into the epidural space after the PTED procedure. To explore the effect of different treatments, a retrospective cohort study was conducted in the Department of Orthopedics of the 907 Hospital of Joint Logistic Support Force. We studied a series of 101 cases of lumbar disc herniation with PTED surgical indications and performed a comparative study dividing the patients into two groups. In group A, each patient received an epidural injection of $40 \mathrm{mg}$ triamcinolone acetonide through the working sleeve following the PTED procedure. In group B, no injection was performed. The differences in efficacy between the two groups were compared.

\section{MATERIAL and METHODS}

This was a retrospective study. Between March 2016 and March 2017, a total of 101 patients diagnosed with singlelevel primary disc herniation who had indications for PTED surgery were retrospectively reviewed. Those patients met the following criteria: they had 1) unilateral radicular pain and 2) single-level primary herniated lumbar disc at the L3/4 level or below. Each patient had symptoms of radicular pain, muscle weakness or numbness of the lower limbs. Either the femoral stretch test or the straight-leg-raising test (Lasegue test) was positive. The clinical symptoms were consistent with the imaging findings. Among the patients, three underwent emergency surgery because of a prolapsed nucleus pulposus in the lumbar disc. Other patients underwent surgery only when radicular pain had lasted more than six weeks and when conservative treatment had been ineffective. The exclusion criteria were sequestered herniation, distally migrated discs, pregnancy, diabetes, coagulopathy, immunocompromise, opioid addiction, infection, spinal canal stenosis, lumbar instability and a history of spinal surgery or epidural injection.

Of 101 patients who met the inclusion criteria, 97 completed a follow-up of longer than two years without recurrence. The patients were divided into two groups based on whether epidural steroids were applied following the PTED procedure. Before September 2016, each patient in group A received an epidural injection of $40 \mathrm{mg}$ triamcinolone acetonide through the working sleeve after PTED. After September 2016, patients in group $B$ were not given the drug.

Before surgery, radiographs were obtained in order to plan for the surgical trajectory, to assess the stability of the lumbar spine and to determine whether there was a high-riding ilium. Computed tomography (CT) scans and magnetic resonance imaging (MRI) were also performed to determine whether there was stenosis of the central canal, a spinal tumor or calcification of the herniated disc.

Informed consent was obtained prior to the intervention. The benefits and risks of the intervention were explained to the patients. The study was approved by the institutional review board.

\section{Description of the Surgical Procedure and Epidural Steroid Application}

The procedure was performed in all patients by an experienced surgeon. Each patient was fixed on an operating table in a lateral position with the affected side directed upwards. Antero-posterior (AP) and lateral fluoroscopy was carried out to locate the superior articular process (SAP) and endplates. The puncture angle and distance from the skin incision to the midline were determined according to the herniated segment, patient size and position of the herniated disc. Therefore, a comprehensive preoperative plan was necessary. Usually, the entry point of L4-5 and L5-S1 is marked $12-14 \mathrm{~cm}$ from the midline, while that of L3-4 is marked $8-10 \mathrm{~cm}$ from the midline. The angle in the craniocaudal direction is commonly $15^{\circ}-30^{\circ}$ for levels L3-4 and L4-5 and is $30^{\circ}-50^{\circ}$ for L5-S1. After sterile draping, the surgical trajectory was infiltrated with local anesthetics, which consisted of a mixture of 10 $\mathrm{ml} 2 \%$ lidocaine, $10 \mathrm{ml} 0.75 \%$ ropivacaine and $15 \mathrm{ml}$ saline. More than $5 \mathrm{ml}$ of the mixture was used at the location of the thoracolumbar fascia. Otherwise, dilation would cause extreme pain at this point. An 18-G needle was introduced towards the SAP. The direction of the needle was checked with $\mathrm{AP}$ and lateral-view fluoroscopy. After the needle tip reached the SAP, the facet joint was anesthetized with the aforementioned mixture. The needle was removed after a guide wire was introduced through it. A skin incision of $7 \mathrm{~mm}$ was made around the guide wire. The soft tissue trajectory was widened with dilation rods. The guide wire was replaced by a Tomshidi needle, which was then tapped through the SAP with its tip approaching the posterior line of the vertebrae with the lateral view and approaching the midline with the AP view. Thereafter, the guide wire was reintroduced to guide the bone drills. The caudal part of the intervertebral foramen was widened step by step with drills of different sizes. A working sleeve was tapped along the dilator, which was inserted into the epidural space immediately after drilling. The C-arm was manipulated to determine whether the working sleeve was in an ideal position. Here after, the endoscope was inserted, and a pressure-regulated pump was connected with the function of rinsing with isotonic solution. Under clear endoscopic view, herniated disc fragments were identified and removed. A disposable bipolar was used to stop local bleeding during the procedure. Decompression was considered to be sufficient only when clear pulsations of the affected nerve root appeared. After the full drainage of isotonic solution in the epidural space, the injection of $40 \mathrm{mg}$ triamcinolone acetonide was performed on each patient in group A. Finally, the incision was sutured after the endoscopic system was retracted from the body.

Patients were suggested to stay in bed for no less than 12 hours after surgery. The same dose of mannitol was applied to promote the regression of edema of the affected nerve root. Ambulatory activities while wearing a brace were allowed the next day. After 48 hours, the patients were encouraged to commence straight leg raising exercises, which reduced the degree of adhesion of the nerve root to the scar tissue. Back muscle exercises were recommended one fortnight after surgery. Neither high-intensity sports nor strenuous labor was allowed within three months. The patients were also advised 
to avoid long periods of sitting or bending, which might result in recurrence.

\section{Efficacy Assessment}

Patients' pain and disability were assessed pre- and postoperatively by using the visual analogue scale (VAS) and Oswestry Disability Index (ODI). VAS scores were obtained one day before surgery and one day, one week, one month, three months, one year and two years postoperatively. ODI scores were collected before surgery and at three months, one year and two years of follow-up.

1. The VAS was used to assess the magnitudes of patients' radicular pain. Zero represents no pain, while ten indicates the most-severe pain.

2. The ODI was applied to assess the degree of disability and pain. It consists of ten items: pain intensity, self-care, lifting, walking, sitting, standing, interfering with sleep, sex life, social life, and tourism. Each item has six options and is scored from zero to five. The sum of scores is expressed as a percentage. The higher the percentage is, the more severe the degree of disability.

3. Patients' satisfaction was assessed after two years using the modified MacNab criteria, which involves four grades. "Excellent" indicates no pain and no restriction of mobility. Patients could return to normal work and activity. "Good" means occasional nonradicular pain and relief of presenting symptoms. Patients could return to modified work. "Fair" signifies some improved functional capacity, and patients are still handicapped and/or unemployed. "Poor" indicates continued objective symptoms of root involvement.

\section{Statistical Analysis Methods}

Statistical analysis was carried out with SPSS version 19.0 software (SPSS, Chicago, IL) and Prism 5.0 (Graph Pad Software Inc., San Diego, CA). Statistical continuous variables were recorded as the mean \pm SD. Between-group comparisons were made using independent t-tests. Categorical variables were analyzed with Fisher's exact test or with a (chi)-squared test. The Mann-Whitney $U$ test for nonparametric data was used for the comparison of over all satisfaction in the two groups. For each analysis, a $p$ value lower than 0.05 was considered statistically significant.

\section{RESULTS}

Of 101 patients who met the inclusion criteria, 97 completed a follow-up of longer than two years without recurrence. Revision surgery was required because of recurrence in 3 patients at 7,16 and 51 weeks. The other patient was lost to follow-up. The average follow-up period was 28.9 months (range, 24-36 months). There were 56 patients in group $A$ and 41 patients in group $B$. The baseline patient characteristics are shown in Table I. There was no significant difference in age, sex, BMI, smoking status or lesion segment between the groups ( $p>0.05)$. No major surgical complications, such as nerve root injury, dural tear, epidural hematoma, vascular injury, abdominal organ injury, discitis or retroperitoneal hematoma, were recorded in either group. Postoperatively, three patients ( 1 in group $A$ and 2 in group $B$ ) presented with transient dysesthesia of the affected lower limbs, which was relieved within one month by conservative treatment.

The surgical procedures of the two groups were basically the same. The average operative time was $85.7 \mathrm{~min}$ (range, 50$143 \mathrm{~min}$ ). The average blood loss during surgery was $41 \mathrm{ml}$ (range, 25-88 ml). The average duration of hospital stay was $5.0 \pm 0.2$ days in group $A$ and $4.8 \pm 0.3$ days in group $B$. The difference was not statistically significant $(p=0.6056)$.

The preoperative VAS score of radicular pain was 6.79 \pm 0.16 in group $A$ and $7.09 \pm 0.23$ in group $B$. Though the latter was slightly higher than the former, the difference was not statistically significant $(p=0.2439)$. The preoperative ODI scores of the group A and group B were $54.4 \pm 1.4$ and

Table I: Baseline Participant Characteristics of Group A and Group B

\begin{tabular}{llll}
\hline & Group A & Group B & p \\
\hline $\mathrm{N}$ & 56 & 41 & \\
\hline Male (\%) & $35(62.5)$ & $23(56.1)$ & $0.5252^{\mathrm{a}}$ \\
\hline Age, years, mean (SD) & $44.6(2.2)$ & $48.9(2.3)$ & $0.1915^{\mathrm{a}}$ \\
\hline BMI, mean (SD) & $22.89(0.29)$ & $23.37(0.34)$ & $0.2967^{\mathrm{a}}$ \\
\hline Smokers (\%) & $22(39.3)$ & $17(41.5)$ & $0.8289^{\mathrm{b}}$ \\
\hline Levels treated & & & $0.7435^{\mathrm{b}}$ \\
\hline L3/4 & 8 & 7 & \\
\hline L4/5 & 29 & 18 & \\
\hline L5/S1 & 19 & 16 & $0.6056^{\mathrm{a}}$ \\
\hline Length of hospital stay (SD) & $5.0(0.2)$ & $4.8(0.3)$ &
\end{tabular}

BMI: Body mass index. 'Students' t-test, ' ${ }^{b}$ hi-square test. 
$55.7 \pm 2.0$, respectively. The difference was too small to be meaningful ( $p=0.5950)$ (Table II).

Postoperative radicular pain and other symptoms were remarkably alleviated in both groups. The VAS scores and ODI scores at each postoperative follow-up point were significantly lower than the preoperative values $(p<0.0001)$. However, there were no statistically significant between-group differences in VAS scores and ODI scores ( $p>0.05)$.

The secondary outcomes of the changes in VAS and ODI scores were also equivalent between the two groups at the 3-month and 2-year follow-ups. At the 3-month follow-up, the changes in the VAS scores of group A and group B were $4.98 \pm 0.21$ and $5.22 \pm 0.29$, respectively, and the changes in the ODI scores were $44.5 \pm 1.5$ and $45.2 \pm 2.0$, respectively. The differences between the two groups were not significant $(p=0.5028,0.7807)$. Similarly, the changes in the VAS and ODI scores at the 2-year follow-up were indistinguishable in the two cohorts $(p=0.5363,0.4932)$ (Figure 1, 2). Regarding the modified MacNab criteria, no significant difference was observed 2 years after treatment between the groups $(p=0.7715)$ (Table III).

Table II: The Comparison of Outcome Measurements between Groups

\begin{tabular}{|c|c|c|c|}
\hline & Group A & Group B & $\mathbf{p}$ \\
\hline \multicolumn{4}{|l|}{ Baseline } \\
\hline VAS (SD) & $6.79(0.16)$ & $7.10(0.23)$ & $0.2439^{*}$ \\
\hline ODI (SD) & $54.4(1.4)$ & $55.7(2.0)$ & $0.5950^{*}$ \\
\hline \multicolumn{4}{|l|}{1 week } \\
\hline VAS (SD) & $2.05(0.10)$ & $2.12(0.10)$ & $0.6450^{*}$ \\
\hline \multicolumn{4}{|l|}{1 month } \\
\hline VAS (SD) & $1.89(0.14)$ & $1.85(0.15)$ & $0.8518^{*}$ \\
\hline \multicolumn{4}{|l|}{3 months } \\
\hline VAS (SD) & $1.80(0.16)$ & $1.88(0.19)$ & $0.7637^{*}$ \\
\hline ODI (SD) & $10.0(0.5)$ & $10.6(0.8)$ & $0.5305^{*}$ \\
\hline \multicolumn{4}{|c|}{ 3-month changes } \\
\hline VAS (SD) & $4.98(0.21)$ & $5.22(0.29)$ & $0.5028^{*}$ \\
\hline ODI (SD) & $44.5(1.5)$ & $45.2(2.0)$ & $0.7807^{*}$ \\
\hline \multicolumn{4}{|l|}{1 year } \\
\hline VAS (SD) & $1.70(0.12)$ & $1.83(0.16)$ & $0.4977^{*}$ \\
\hline ODI (SD) & $9.2(0.5)$ & $9.5(0.7)$ & $0.7791^{*}$ \\
\hline \multicolumn{4}{|l|}{2 years } \\
\hline VAS(SD) & $1.46(0.11)$ & $1.59(0.13)$ & $0.4743^{*}$ \\
\hline ODI (SD) & $8.8(0.5)$ & $8.3(0.7)$ & $0.5963^{*}$ \\
\hline \multicolumn{4}{|c|}{ 2-year changes } \\
\hline VAS(SD) & $5.32(0.19)$ & $5.51(0.25)$ & $0.5363^{*}$ \\
\hline ODI (SD) & $45.7(1.4)$ & $47.4(2.2)$ & $0.4932^{*}$ \\
\hline
\end{tabular}

VAS: Visual analogue scale for radicular pain, ODI: Oswestry disability index. * Student's t-test.

Table III: The Comparison of Satisfaction between Groups at the 2-Year Follow-Up Using the Modified MacNab Criteria

\begin{tabular}{ccccc}
\hline & Excellent & Good & Fair & Poor \\
\hline Group A & 40 & 11 & 3 & 2 \\
\hline Group B & 27 & 10 & 3 & 1 \\
\hline
\end{tabular}

p: 0.7715 using the Mann-Whitney $U$ test. 


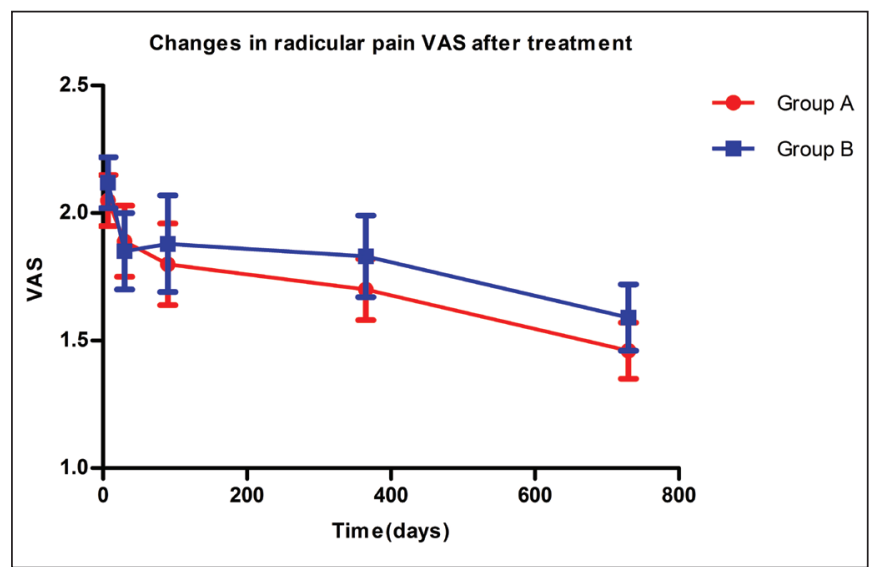

Figure 1: Changes in the VAS scores 1 week, 1 month, 3 months, 1 year and 2 years after treatment.

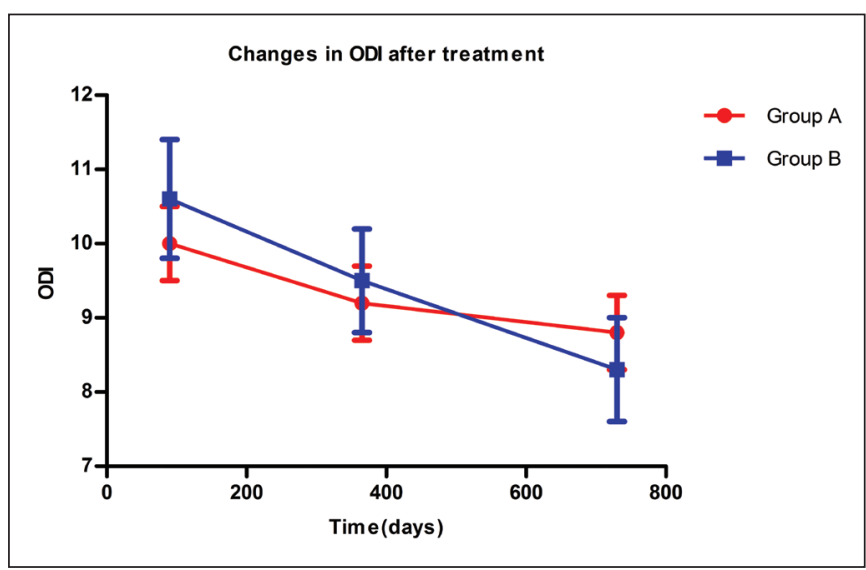

Figure 2: Changes in the ODI scores 3 months, 1 year and 2 years after treatment.

\section{DISCUSSION}

PTED, the least-invasive type of disc surgery, has obvious advantages over traditional open discectomy. To some extent, PTED is even better than MED because it has the merits of causing less intraoperative blood loss, having a smaller risk of infection and so on $(3,5,16)$. In addition, lumbar MED surgery is performed under epidural/general anesthesia, while PTED only requires local anesthesia. Therefore, anesthesia contraindications for surgery are rare. In addition, during the procedure, surgeons can communicate with patients at any time to obtain feedback. Thus, the possibility of iatrogenic nerve root injury can be greatly reduced. Finally, the skin incision size required for PTED is only $7 \mathrm{~mm}$. The damage to soft tissue is mild, and the amount of blood loss is small. In this study, the average amount of intraoperative blood loss was 41 $\mathrm{ml}$ (range, 25-88 ml). PTED can avoid the paraspinal muscle denervation and atrophy caused by excessive dissection and traction in traditional surgery. Thus, the procedure reduces the occurrence of failed back surgery syndromes, which have a relatively high incidence after traditional surgery (8). The procedure also causes limited damage to bone structure.
Only the ventral side of the superior articular process of the underlying vertebra is removed during foraminoplasty, which causes no change in the biomechanical environment postoperatively according to some related studies $(17,23)$.

Moreover, under the magnifying effect of the endoscope and video system, anatomical structures such as the nucleus pulposus and nerve roots can be clearly distinguished. Therefore, the incidence of iatrogenic nerve root injury can be further decreased. In this study, no patients had intraoperative complications such as nerve root injury or dural tear. The use of an endoscope also enables surgeons to remove disc fragments easily and release the affected nerve root adequately. The exiting nerve root and dural sac are well protected from irritation during the procedure. Bleeding can be stopped effectively by a trigger-flex bipolar radio frequency. Therefore, the risk of postoperative epidural hematoma can be lessened.

Minimal surgical trauma enables quick postoperative rehabilitation and a corresponding reduction in the duration of hospital stay. In this study, the average duration of hospital stay was $5.0 \pm 0.2$ days in group $A$ and $4.8 \pm 0.3$ days in group B. Because of the favorable clinical outcomes and low readmission rates, it is widely known that percutaneous transforaminal endoscopic discectomy is carried out in ambulatory surgery centers $(7,11,15)$.

In clinical practice, there are numerous patients with lumbar disc herniation. Sometimes, severe disc herniation does not lead to the corresponding degree of radicular pain. It is worth noting that the clinical symptoms of some patients arenot significantly alleviated even after sufficient surgical decompression. This is because mechanical nerve root compression is not the only cause of pain, although it is the most common cause $(14,22)$.

It has been suggested that the epidural application of autologous nucleus pulposus without mechanical compression causes nerve root inflammation and related radicular pain in lumbar disc herniation. The nucleus pulposus contains chemical compositions that can irritate nerve roots. Inflammatory mediators can induce a series of chemical modulator interactions. Then, inflammatory cascade reactions occur. A nerve root with inflammation is extremely sensitive to stimulations such as pressure and stretch. Ache signals are transmitted continuously, and pain is there by produced (19). Hence, a rationale for the use of epidural corticosteroids to inhibit inflammation and alleviate pain can be formed. Transforaminal epidural steroid injection is one of the most common nonsurgical treatments prescribed for radicular pain caused by lumbar disc herniation. Although corticosteroids have been shown to play an anti-inflammatory role by inhibiting either the synthesis or release of a number of proinflammatory mediators and by forming a reversible local anesthetic effect, the mechanisms remain unproven $(4,13,18)$.

Previous reports on the efficacy of epidural steroid injection are inconsistent. Taskaynatan et al.'s study included 80 patients who received fluoroscopically guided transforaminal epidural steroid injections performed by the same physician for chronic 
radicular low back pain caused by lumbar disc herniation. The mean duration of the injection effect was $12.46 \pm 7.24$ months. The response rate for the epidural steroid injections was $72 \%$. These authors considered transforaminal epidural steroid injections as an alternative treatment for managing chronic radicular low back pain (20). In a retrospective study involving 367 patients, Berman et al. reported that although the efficacy of epidural injection declined over time, $60 \%$ of patients still achieved good results within one year (2). Watts et al. conducted a meta-analysis of epidural steroid injection for the treatment of sciatica. The analysis, which involved 11 randomized controlled trials and 907 patients, showed that epidural administration of corticosteroids was effective in the management of lumbosacral radicular pain, although the efficacy decreased over time (21).

Corticosteroids are classified as particulate or nonparticulate formulations. Triamcinolone, methylprednisolone and betamethasone are representative of particulate corticosteroids, while dexamethasone belongs to the nonparticulate category. The variety of corticosteroids that can be selected for epidural injection is also controversial. Bensler et al. compared particulate with nonparticulate corticosteroids in a retrospective study. The results showed that the effectiveness of particulate corticosteroids was better than that of nonparticulate corticosteroids both 1 week and 1 month post injection. The difference was statistically significant. In particular, the outcomes of patients who received nonparticulate corticosteroids worsened after the first week (1). Kim et al. performed an intraindividual comparison of the effectiveness of lumbar epidural steroid injection between triamcinolone and dexamethasone. The relative satisfaction with the former type of steroid was significantly better than that with the latter type of steroid in the same patient (10). By contrast, Kennedy et al. conducted a multicenter, double blind, prospective, randomized trial of 78 consecutive patients with unilateral radicular pain due to acute unilevel disc herniation. In that trial, the effectiveness of dexamethasone was shown to be noninferior to that of triamcinolone (9). Even in the same category, triamcinolone and betamethasone had different levels of effectiveness. In the longitudinal cohort study of McCormick et al., 1568 patients with lumbosacral radicular pain accepted transforaminal epidural steroid injections with triamcinolone or betamethasone. More frequent pain relief of greater than $50 \%$ at the short-term follow-up was reported with triamcinolone (12).

In this study, additional epidural injection of triamcinolone was administered into the epidural space and around the nerve roots to further improve the clinical efficacy. However, the study showed that no additional effectiveness of epidural steroid injection following PTED was observed. There were no significant differences in the postoperative VAS and ODI scores between the two groups at each follow-up. The results suggest that, for patients with lumbar disc herniation who have clear indications for surgery, the key is to remove the herniated disc fragments that exert physical compression and chemical stimulation on the corresponding nerve roots. Although epidural steroid injection may promote the regression of inflammation and alleviate radicular pain, the additional effectiveness is not significant.

There are several weaknesses of the study. First, it is a retrospective study rather than a randomized controlled trial. However, there were no significant between-group differences in the baseline characteristics. Second, data reflecting the degree of back pain were not collected. Third, health-related quality of life, which is usually measured by the 36-item Short Form (SF-36) or 12-item Short Form, was not evaluated during the follow-up. A further limitation is the lack of data on postoperative analgesic use, which would inevitably have influenced the VAS and ODI scores. Hence, a large-sample, double-blind, randomized controlled trial is required in future studies.

\section{CONCLUSION}

PTED is a safe and effective minimally invasive surgery for radiculopathy caused by lumbar disc herniation. Epidural steroid injection following the PTED procedure offered no benefit compared with PTED surgery alone. Therefore, the additional application of epidural steroids might not be necessary.

\section{REFERENCES}

1. Bensler S, Sutter R, Pfirrmann CWA, Peterson CK: Particulate versus non-particulate corticosteroids for transforaminal nerve root blocks: Comparison of outcomes in 494 patients with lumbar radiculopathy. Eur Radiol 28:946-952, 2018

2. Berman AT, Garbarino JL Jr, Fisher SM, Bosacco SJ: The effects of epidural injection of local anesthetics and corticosteroids on patients with lumbosciatic pain. Clin Orthop Relat Res 188:144-151, 1984

3. Choi KC, Shim HK, Kim JS, Cha KH, Lee DC, Kim ER, Kim MJ, Park CK: Cost-effectiveness of microdiscectomy versus endoscopic discectomy for lumbar disc herniation. Spine J, 2019

4. Dietrich CL, Smith CE: Epidural granuloma and intracranial hypotension resulting from cervical epidural steroid injection. Anesthesiology 100:445-447, 2004

5. Gibson JNA, Subramanian AS, Scott CEH: A randomised controlled trial of transforaminal endoscopic discectomy vs microdiscectomy. Eur Spine J 26:847-856, 2017

6. Hayashi N, Weinstein JN, Meller ST, Lee HM, Spratt KF, Gebhart GF: The effect of epidural injection of betamethasone or bupivacaine in a rat model of lumbar radiculopathy. Spine (Phila Pa 1976) 23:877-885, 1998

7. Idowu OA, Boyajian HH, Ramos E, Shi LL, Lee MJ: Trend of spine surgeries in the outpatient hospital setting versus ambulatory surgical center. Spine (Phila $\mathrm{Pa}$ 1976) 42:E1429-E1436, 2017

8. Kapural L, Peterson E, Provenzano DA, Staats P: Clinical evidence for spinal cord stimulation for failed back surgery syndrome (fbss): Systematic review. Spine (Phila Pa 1976) 42 Suppl 14:S61-S66, 2017 
LV J. et al: Percutaneous Transforaminal Endoscopic Discectomy

9. Kennedy DJ, Plastaras C, Casey E, Visco CJ, Rittenberg JD, Conrad B, Sigler J, Dreyfuss P: Comparative effectiveness of lumbar transforaminal epidural steroid injections with particulate versus nonparticulate corticosteroids for lumbar radicular pain due to intervertebral disc herniation: A prospective, randomized, double-blind trial. Pain Med 15:548555,2014

10. Kim JY, Lee JW, Lee GY, Lee E, Yoon CJ, Kang HS: Comparative effectiveness of lumbar epidural steroid injections using particulate vs. non-particulate steroid: An intra-individual comparative study. Skeletal Radiol 45:169-176, 2016

11. Lewandrowski KU: Incidence, management, and cost of complications after transforaminal endoscopic decompression surgery for lumbar foraminal and lateral recess stenosis: A value proposition for outpatient ambulatory surgery. Int $\mathrm{J}$ Spine Surg 13:53-67, 2019

12. McCormick Z, Kennedy DJ, Garvan C, Rivers E, Temme K, Margolis S, Zander E, Rohr A, Smith MC, Plastaras C: Comparison of pain score reduction using triamcinolone vs. betamethasone in transforaminal epidural steroid injections for lumbosacral radicular pain. Am J Phys Med Rehabil 94:1058-1064, 2015

13. McLain RF, Kapural L, Mekhail NA: Epidural steroid therapy for back and leg pain: Mechanisms of action and efficacy. Spine J 5:191-201, 2005

14. Olmarker K: Spinal nerve root compression. Nutrition and function of the porcine cauda equina compressed in vivo. Acta Orthop Scand Suppl 242:1-27, 1991

15. Pak LM, Fogel HA, Chaudhary MA, Kwon NK, Barton LB, Koehlmoos T, Haider AH, Schoenfeld AJ: Outpatient spine clinic utilization is associated with reduced emergency department visits following spine surgery. Spine (Phila $\mathrm{Pa}$ 1976) 43:E836-E841, 2018
16. Sairyo K, Egawa H, Matsuura T, Takahashi M, Higashino K, Sakai T, Suzue N, Hamada D, Goto T, Takata Y, Nishisho T, Goda Y, Sato R, Tsutsui T, Tonogai I, Kondo K, Tezuka F, Mineta K, Sugiura K, Takeuchi M, Dezawa A: State of the art: Transforaminal approach for percutaneous endoscopic lumbar discectomy under local anesthesia. J Med Invest 61: 217-225, 2014

17. Sairyo K, Goel VK, Masuda A, Biyani A, Ebraheim N, Mishiro T, Terai T: Biomechanical rationale of endoscopic decompression for lumbar spondylolysis as an effective minimally invasive procedure - a study based on the finite element analysis. Minim Invasive Neurosurg 48:119-122, 2005

18. Tachihara H, Sekiguchi M, Kikuchi S, Konno S: Do corticosteroids produce additional benefit in nerve root infiltration for lumbar disc herniation? Spine (Phila Pa 1976) 33:743-747, 2008

19. Takebayashi T, Cavanaugh JM, Cuneyt Ozaktay A, Kallakuri S, Chen C: Effect of nucleus pulposus on the neural activity of dorsal root ganglion. Spine (Phila Pa 1976) 26:940-945, 2001

20. Taskaynatan MA, Tezel K, Yavuz F, Tan AK: The effectiveness of transforaminal epidural steroid injection in patients with radicular low back pain due to lumbar disc herniation two years after treatment. J Back Musculoskelet Rehabil 28:447451, 2015

21. Watts RW, Silagy CA: A meta-analysis on the efficacy of epidural corticosteroids in the treatment of sciatica. Anaesth Intensive Care 23:564-569, 1995

22. Winkelstein BA, DeLeo JA: Mechanical thresholds for initiation and persistence of pain following nerve root injury: Mechanical and chemical contributions at injury. J Biomech Eng 126:258263, 2004

23. Yokosuka J, Oshima Y, Kaneko T, Takano Y, Inanami H, Koga $\mathrm{H}$ : Advantages and disadvantages of posterolateral approach for percutaneous endoscopic lumbar discectomy. J Spine Surg 2:158-166, 2016 